\title{
Luminosity determination for the quasi-free nuclear reactions
}

\author{
P. Moskal and R. Czyżykiewicz for the COSY-11 collaboration \\ Institute of Physics, Jagellonian University, 30-059 Cracow, Poland \& \\ Institut für Kernphysik, Forschungszentrum Jülich, 52425 Jülich, Germany
}

\begin{abstract}
A method for the calculation of the luminosity for the proton-nucleus collisions based on the quasi-free proton-proton scattering is presented. As an example of application the integrated luminosity for the scattering of protons off the deuteron target is determined for the experiment of the quasi-free $p n \rightarrow p n \eta$ reaction performed by means of the COSY-11 facility.
\end{abstract}

Keywords: luminosity, quasi-free meson production, $\eta$ meson production

PACS: 14.40.Aq, 13.60.Le

\section{INTRODUCTION}

Due to the lack of the pure neutron targets a meson production in the proton-neutron reactions is usually realised e.g. via the proton scattering off the deuteron. This method takes advantage of the fact that the neutron's binding energy inside the deuteron is negligible relative to the kinetic energy of proton beams needed to produce the meson in the nucleon-nucleon collisions [1, 2]. In the analysis of such reactions it is assumed that the proton is acting only as a spectator and that it is on its mass shell at the moment of the collision.

In this contribution we present the evaluation of the luminosity in such kind of experiments by means of the measurement of the quasi-free proton-proton scattering. This method permits to take into account automatically the shadowing effects of the spectator nucleon, and allows for the determination of the luminosity with a relatively small normalization uncertainty thanks to the availability of the precise cross sections for the proton-proton elastic scattering determined by the EDDA collaboration [3] up to the beam momentum of $3.5 \mathrm{GeV} / \mathrm{c}$.

\section{LUMINOSITY}

In order to determine the luminosity for the quasi-free proton-neutron reaction we measured quasi-free $p p \rightarrow p p$ reaction by detecting in coincidence both scattered protons. Here we will describe the measurement performed by means of the COSY-11 apparatus [4, 5, 6, 7] shown schematically in Figure 1 (left). The recoil proton gives signal in the scintillator detector S4 and subsequently reaches the granulated silicon detector $\mathrm{Si}_{\text {mon }}$, while the forward scattered proton is registered by the stack of drift chambers D1 and D2 and scintillator array S1. For triggering of the elastic scattered events the coincidence between signals from the S1 and S4 scintillators was required. 

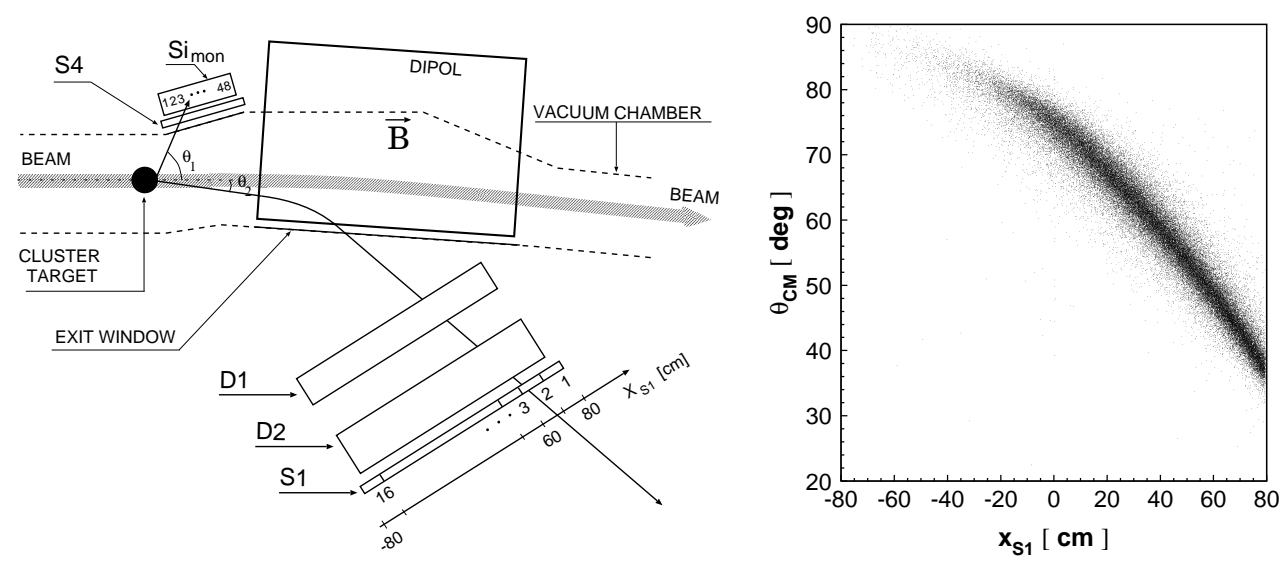

FIGURE 1. (left) Schematic view of the COSY-11 detection setup. Only the detectors used for the measurements of the quasi-free proton-proton scattering are shown. D1 and D2 denotes the stack of two drift chambers. $\mathrm{Si}_{\text {mon }}$ is the granulated silicon detector. $\mathrm{S} 1$ and $\mathrm{S} 4$ stand for the scintillator detectors. (right) Relation between the center-of-mass scattering angle and the position in the S1 detector for the quasi-free proton-proton scattering at $\mathrm{p}_{\text {beam }}=2.075 \mathrm{GeV} / \mathrm{c}$ as has been obtained in the Monte-Carlo simulations.

In the case of free proton-proton scattering the luminosity could be determined as a normalization constant between the measured angular distribution of the cross section and the corresponding spectrum known from the previous experiments. For the quasifree reaction the evaluation becomes more complicated due to the Fermi motion of nucleons inside the nucleus (Fig. 2 (left)). Since the direction and momentum of the bound nucleon may vary from event to event this implies that the direction of the center-of-mass velocity of the colliding nucleons as well as the total available energy for the reaction may also vary from event to event. Therefore, protons registered in the laboratory under a given scattering angle or at a given part of the detection system, correspond to the finite range of scattering angles in the proton-proton center-of-mass frame (see Fig. 11(right)). This implies that the experimental angular distributions cannot be directly compared to the literature values, and instead an evaluation of the luminosity requires simulations taking into account the Fermi motion of the nucleons, and the variations of differential cross sections for the elastic scattering as a function of the scattering angle and energy.

For each simulated event we know the generated Fermi momentum of the nucleon, as well as the scattering angle of protons in their center-of-mass system. This permits us to assign to each event a weight corresponding to the differential cross section, which is uniquely determined by the scattering angle and the total collision energy $s$.

For the intuitive illustration of the size of the momentum spread caused by the Fermi motion instead of the total center-of-mass energy we may equivalently consider the effective beam momentum as it is seen from the nucleon inside the nucleus. The distribution of the effective beam momentum depends on the value of the proton beam momentum and as an example in Figure 2 (middle) we present it for the value of $2075 \mathrm{MeV} / \mathrm{c}$ as which used for the measurement of the quasi free $p n \rightarrow p n \eta$ reaction [8, 9]. It is important to note that the distribution of the equivalent beam momentum ranges 

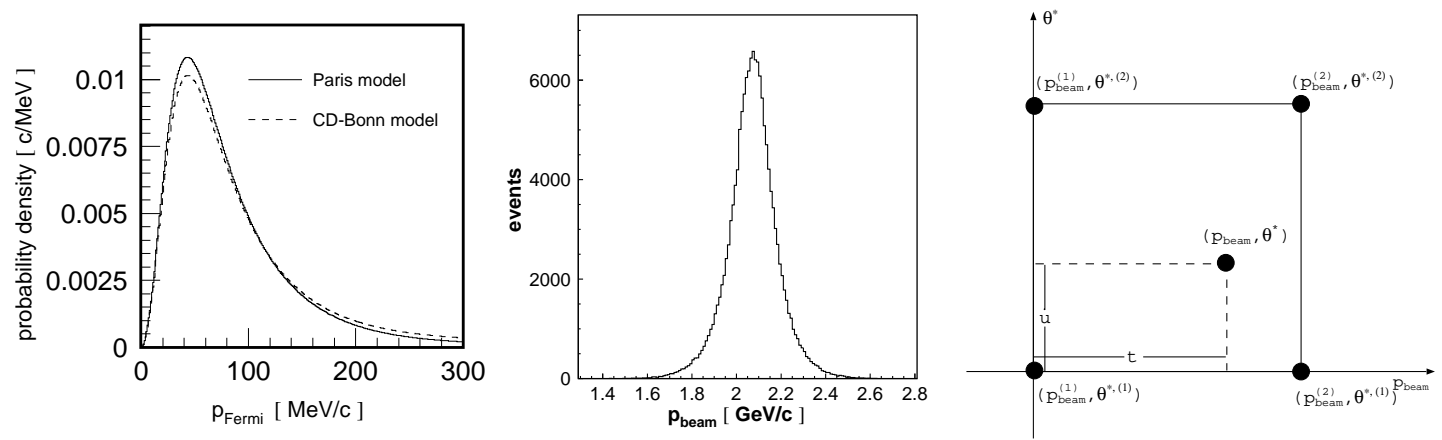

FIGURE 2. (left panel) Nucleon momentum distribution inside the deuteron according to the Paris [10] (full line) and CDBONN [11] (dotted line) potentials. (middle panel) Distribution of the beam momentum as seen by the proton bounded inside a deuteron hit by the beam proton with momentum of $p_{\text {beam }}=2.075 \mathrm{GeV} / \mathrm{c}$. (right) Bilinear interpolation of the differential cross section $\frac{d \sigma}{d \Omega}\left(p_{\text {beam }}, \theta^{*}\right)$.

from about $1.5 \mathrm{GeV} / \mathrm{c}$ up to circa $2.5 \mathrm{GeV} / \mathrm{c}$. In this momentum range the cross sections for the proton-proton elastic scattering vary significantly [3] and therefore this effect cannot be neglected.

In the following we will be more specific and will describe the derivation of the luminosity in terms of formulae exploited in the analysis. For a free proton-proton scattering we could measure the number of events $-\Delta N(\theta, \phi)$ scattered into the solid angle $\Delta \Omega(\theta, \phi)$ around the polar and azimuthal angles $\theta$ and $\phi$, respectively. In this case the angles in laboratory and in the center-of-mass systems are univocally related to each other. With the known differential cross section [3] for proton-proton scattering into that particular solid angle, and having known the value of the solid angle $\Delta \Omega(\theta, \phi)$ from the Monte-Carlo simulations the luminosity can be calculated according to the formula:

$$
L=\frac{\Delta N(\theta, \phi)}{\Delta \Omega(\theta, \phi) \frac{d \sigma}{d \Omega}(\theta, \phi)} .
$$

In the case of quasi-free proton-proton scattering the number of elastically scattered protons $\Delta N$ into a solid angle $\Delta \Omega\left(\theta_{l a b}, \phi_{l a b}\right)$ is proportional to $L$ - the integrated luminosity over the time of measurement, and also to the inner product of the differential cross section for scattering into the solid angle around $\theta^{*}$ and $\phi^{*}$ angles $\frac{d \sigma}{d \Omega}\left(\theta^{*}, \phi^{*}, p_{F}, \theta_{F}, \phi_{F}\right)$ - and the probability density of the distribution of the Fermi momentum $f\left(p_{F}, \theta_{F}, \phi_{F}\right)$ :

$$
\begin{gathered}
\Delta N_{\exp }\left(\Delta \Omega\left(\theta_{l a b}, \phi_{l a b}\right)\right)= \\
L \int_{\Delta \Omega\left(\theta_{l a b}, \phi_{l a b}\right)} \frac{d \sigma}{d \Omega}\left(\theta^{*}, \phi^{*}, p_{F}, \theta_{F}, \phi_{F}\right) f\left(p_{F}, \theta_{F}, \phi_{F}\right) d p_{F} d \cos \theta_{F} d \phi_{F} d \phi^{*} d \cos \theta^{*}
\end{gathered}
$$

The angles $\theta^{*}$ and $\phi^{*}$ are expressed in the proton-proton center-of-mass system, while the angles $\theta_{l a b}$ and $\phi_{l a b}$ are considered in the laboratory system. In the case of the complex detection geometry with a magnetic field a solid angle corresponding to a given part of the detector cannot in general be expressed in a closed analytical form. 
Therefore, integral in Equation 2 must be computed using the Monte-Carlo simulation programme, containing the exact geometry of the detection system and taking into account bending of particles trajectories in the magnetic field as well as detection and reconstruction efficiencies. For the evaluation of a given event by the Monte-Carlo programme first we choose randomly a momentum of a nucleon inside a deuteron according to the Fermi distribution [10] (Fig. 2 (left)). Next the total energy $s$ for the proton-proton scattering and the vector of the center-of-mass velocity are determined. Then, we generate isotropically a momentum of protons in the proton-proton centerof-mass frame. Further on, according to the generated angle and the total-energy $s$ (or equivalently an effective beam momentum seen by the struck nucleon) we assign to the event a probability equal to the differential cross section. Next, the momenta of protons are transformed to the laboratory frame and are used as an input in the simulation of the detectors signals with the use of the GEANT computing package.

The differential cross sections $\frac{d \sigma}{d \Omega}\left(\theta^{*}, \phi^{*}, s\right)$, with $s$ being dependent on $p_{F}, \theta_{F}, \phi_{F}$, and $p_{\text {beam }}$, and with $\theta^{*}$ and $\phi^{*}$ denoting the scattering angles in the proton-proton center-of-mass system were calculated using the cross section data base for the $p p \rightarrow$ $p p$ reaction [3] 1. For this purpose we have applied a bilinear interpolation in the momentum-scattering angle plane and calculated differential cross section according to the formula:

$$
\begin{array}{r}
\frac{d \sigma}{d \Omega}\left(p_{\text {beam }}, \theta^{*}\right)=(1-t)(1-u) \frac{d \sigma}{d \Omega}\left(p_{\text {beam }}^{1}, \theta^{*, 1}\right)+t(1-u) \frac{d \sigma}{d \Omega}\left(p_{\text {beam }}^{2}, \theta^{*, 1}\right)+ \\
t u \frac{d \sigma}{d \Omega}\left(p_{\text {beam }}^{2}, \theta^{*, 2}\right)+(1-t) u \frac{d \sigma}{d \Omega}\left(p_{\text {beam }}^{1}, \theta^{*, 2}\right),
\end{array}
$$

where variables $t$ and $u$ are defined in Figure 2 (right). These cross sections were used as the weights of the elastically scattered events in the Monte-Carlo calculations.

$\Delta N_{\text {exp }}$ from Equation 2 can be determined as a number of elastically scattered protons registered in a given part of the detector system. In order to calculate the integral on the right hand side of this equation we simulated $N_{0}$ events according to the procedure described above. Due to the weights assigned to the events the integral is not dimensionless and its units correspond to the units of the cross sections used for the calculations. The number obtained from the Monte-Carlo simulations must be then normalized such that the integral over the full solid angle equals to the total cross section for the elastic scattering averaged over the distribution of the total reaction energy $s$ resulting from the Fermi distribution of the target nucleon. In the absence of the Fermi motion it should be simply equal to a total elastic cross section for a given beam momentum. This means that we need to divide the resultant integral by the number of generated events $N_{0}$ and multiply it by the factor of $2 \pi$. A factor $2 \pi$ comes from the normalization of the differential cross section $\frac{d \sigma}{d \Omega}\left(p_{\text {beam }}, \theta^{*}, \phi^{*}\right)$, regarding the fact that protons taking part in the scattering are indistinguishable. Hence, the formula for the calculation of the integrated

${ }^{1}$ EDDA group has gathered the data for the excitation functions $\frac{d \sigma}{d \Omega}\left(\theta^{*}, p_{\text {beam }}\right)$ for the elastic $p p \rightarrow$ $p p$ process at 108 different proton kinetic energies, ranging from $240 \mathrm{MeV}$ up to $2577 \mathrm{MeV}$. In the measurements the center-of-mass scattering angles of protons $\left(\theta^{*}\right)$ from $30^{\circ}$ up to $90^{\circ}$ have been covered. Both the kinetic and angular ranges are sufficient to cover our needs in calculating the corresponding differential cross sections. 
luminosity for the quasi-free reaction reads:

$$
L=\frac{N_{0} \Delta N_{\text {exp }}}{2 \pi \int_{\Delta \Omega\left(\theta_{l a b}, \phi_{l a b}\right)} \frac{d \sigma}{d \Omega}\left(\theta^{*}, \phi^{*}, p_{F}, \theta_{F}, \phi_{F}\right) f\left(p_{F}, \theta_{F}, \phi_{F}\right) d p_{F} d \cos \theta_{F} d \phi_{F} d \phi^{*} d \cos \theta^{*}}
$$

where the normalization constant $N_{0} / 2 \pi$ is subject to the Monte-Carlo method used for the integral computation.

\section{EXAMPLE OF APPLICATION}

We have applied the above described method for the evaluation of the luminosity for the measurement of the $p n \rightarrow p n \eta$ reaction with the COSY-11 facility [8, 9, 13].

Events corresponding to the elastically scattered protons have been identified on the basis of the momentum distributions. The momentum of the fast scattered proton, whose trajectory has been registered in the drift chambers, can be reconstructed, and the transversal versus the parallel momentum component may be plotted as it is done in left panel of Figure 3, The signal from the elastic scattered protons appears as an clear enhancement around the expected kinematical ellipse.
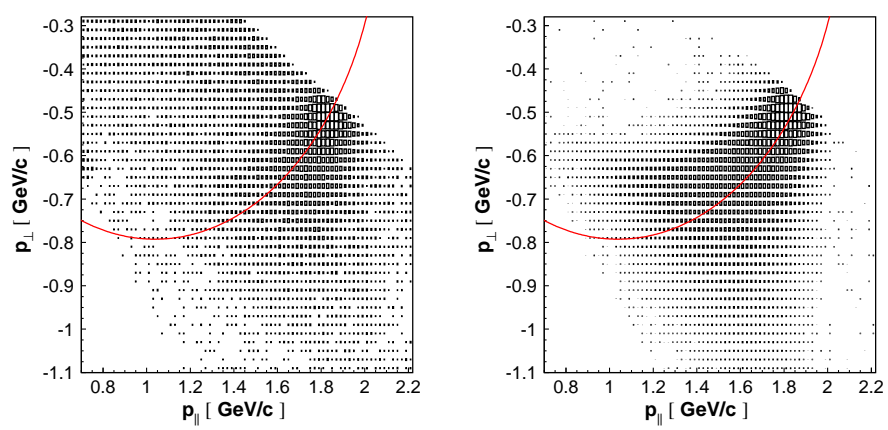

FIGURE 3. Parallel versus transversal momentum component of the reconstructed fast proton momentum as obtained in the experiment (left) and in the simulations (right). Superimposed lines correspond to the expected kinematical ellipses.

For the calculations of the integrated luminosity, the part of the S1 detector available for the elastically scattered protons was divided into four subranges. In order to separate the background from the multi particle reactions for each subrange, the distribution of the distance of the points to the kinematical ellipse was determined. The result obtained for different sections of the S1 detector are presented in Figure 4

A linear background cut 2 has been performed and subsequently the true scattering yields into a given range of the S1 detector have been calculated.

For the determination of the integral of Equation 2 an $\mathrm{N}_{0}=10^{7}$ quasi free $p p \rightarrow p p$ events have been simulated, and the response of the detectors has been generated using

2 The main source of the background are the accidental coincidences originating from the production and scattering processess. 

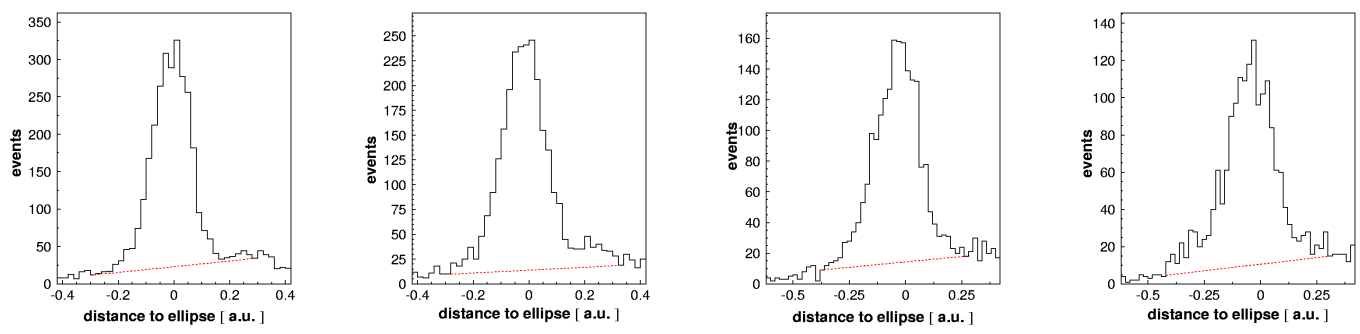

FIGURE 4. Projection along the expected kinematical ellipse of the experimental event distribution from Figure 3 (left) for four subranges of the S1 detector.
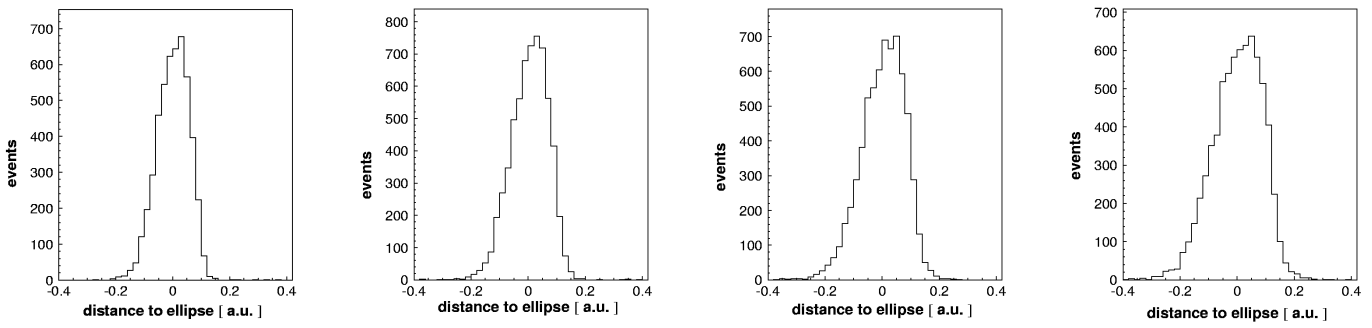

FIGURE 5. Projection along the expected kinematical ellipse of the simulated event distribution from Figure 3 (right) for four subranges of the $\mathrm{S} 1$ detector.

the GEANT-3 code based simulation programme, maintaining the experimental conditions of beam and target [14]. Subsequently, the simulated events have been analyzed in the same way as the experimental data. Figure 5 shows the simulated spectra analogous to the experimental distributions of Figure 4. As described in the previous sections each entry in the shown histograms was weighted according to the differential cross sections and hence the integral of these spectra normalized to number of simulated events and multiplied by a factor of $2 \pi$ can be substituted for an integral in Equation 2, Thus the integrals of experimental (Fig. (4) and simulated histograms (Fig. 5) applied in Equation 2 permitted to determine the luminosity for each of the subrange of S1 detector separately. The weighted average over the four quoted values of the integrated luminosity equals to $\mathrm{L}=(2.08 \pm 0.03) \cdot 10^{35} \mathrm{~cm}^{-2}$.

One source of the systematic error may be attached to the background misidentification. Since the line describing the background can be well defined on both: the left and the right side of the scattering peaks, we assumed, conservatively, that the systematic error due to the assumption of the linearity of the background in the way as presented in Figure 4 is less than $20 \%$. The background events constitute around $15 \%$ of all events, hence the overall systematic error due to the background subtraction is not greater than $3 \%$.

Another source of the systematic error originates from the assumption of the bilinear approximation of the cross section shown in Figure 2(right). To estimate this systematic uncertainty we made the zeroth-order assumption in which instead of the interpolation we took the cross section value from the closest data point in the effective proton beam momentum-scattering angle plane. Higher order approximations of the differential cross 
section given by Equation 3 should be not greater than the difference between the zeroth order approximation explained above and the bilinear approximation. The performed calculations shows that this difference is smaller than $0.2 \%$.

Taking into account the normalisation error of the EDDA differential cross sections (equal to circa 4\% [3, 15]), the systematical error originating from the assumption of the potential model of the nucleon bound inside the deuteron (equal to about $2 \%$ ), and the two abovementioned sources of the systematical errors, we estimated the overall systematical error of the integrated luminosity to be not greater than $9.2 \%$.

\section{ACKNOWLEDGMENTS}

We acknowledge the support of the European Community-Research Infrastructure Activity under the FP6 programme (Hadron Physics, N4:EtaMesonNet, RII3-CT-2004506078), the support of the Polish Ministry of Science and Higher Education under the grants No. PB1060/P03/2004/26, 3240/H03/2006/31 and 1202/DFG/2007/03, and

the support of the German Research Foundation (DFG) under the grant No. GZ: 436 POL 113/117/0-1.

\section{REFERENCES}

1. P. Moskal et al., Prog. Part. Nucl. Phys. 49, 1 (2002).

2. P. Moskal et al., Int. J. Mod. Phys. A22, 305 (2007).

3. D. Albers et al., Phys. Rev. Lett. 78, 1652 (1997).

4. S. Brauksiepe et al., Nucl. Instrum. Meth. A 376, 397 (1996).

5. H. Dombrowski et al., Nucl. Instrum. Meth. A 386, 228 (1997).

6. J. Smyrski et al., Nucl. Instrum. Meth. A 541, 574 (2005).

7. P. Klaja et al., AIP Conf. Proc. 796, 160 (2005).

8. P. Moskal et al., e-Print Archive: nucl-ex/0110001.

9. P. Moskal et al., J. Phys. G 32, 629 (2006).

10. M. Lacombe et al., Phys. Lett. B 101, 139 (1981).

11. R. Machleidt et al., Phys. Rev. C 63, 024001 (2001).

12. P. Moskal, PhD Thesis, Jagiellonian University (1998).

13. J. Przerwa et al., AIP Conf. Proc. 796, 164 (2005).

14. P. Moskal et al., Nucl. Instrum. Meth. A 466, 448 (2001).

15. A. J. Simon et.al., Phys. Rev. C 48, 662 (1993). 